\title{
Article \\ Robotic Replica of a Human Spine Uses Soft Magnetic Sensor Array to Forecast Intervertebral Loads and Posture after Surgery
}

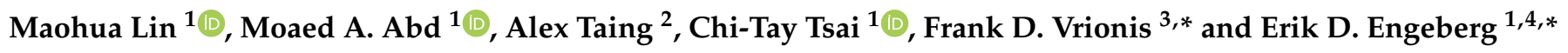 \\ 1 Department of Ocean and Mechanical Engineering, Florida Atlantic University, Boca Raton, FL 33431, USA; \\ mlin2014@fau.edu (M.L.); mabd2015@fau.edu (M.A.A.); tsaict@fau.edu (C.-T.T.) \\ 2 Department of Biomedical Engineering, University of Virginia, Charlottesville, VA 22904, USA; \\ alt6yzw@virginia.edu \\ 3 Department of Neurosurgery, Marcus Neuroscience Institute, Boca Raton Regional Hospital, \\ Boca Raton, FL 33486, USA \\ 4 Center for Complex Systems and Brain Sciences, Florida Atlantic University, Boca Raton, FL 33431, USA \\ * Correspondence: FVrionis@baptisthealth.net (F.D.V.); eengeberg@fau.edu (E.D.E.)
}

check for updates

Citation: Lin, M.; Abd, M.A.; Taing, A.; Tsai, C.-T.; Vrionis, F.D.; Engeberg, E.D. Robotic Replica of a Human Spine Uses Soft Magnetic Sensor Array to Forecast Intervertebral Loads and Posture after Surgery. Sensors 2022, 22, 212. https:// doi.org/10.3390/s22010212

Academic Editors: Shunrou Fujiwara and Kuniaki Ogasawara

Received: 22 November 2021 Accepted: 23 December 2021 Published: 29 December 2021

Publisher's Note: MDPI stays neutral with regard to jurisdictional claims in published maps and institutional affiliations.

Copyright: (C) 2021 by the authors. Licensee MDPI, Basel, Switzerland. This article is an open access article distributed under the terms and conditions of the Creative Commons Attribution (CC BY) license (https:// creativecommons.org/licenses/by/ $4.0 /)$.

\begin{abstract}
Cervical disc implants are conventional surgical treatments for patients with degenerative disc disease, such as cervical myelopathy and radiculopathy. However, the surgeon still must determine the candidacy of cervical disc implants mainly from the findings of diagnostic imaging studies, which can sometimes lead to complications and implant failure. To help address these problems, a new approach was developed to enable surgeons to preview the post-operative effects of an artificial disc implant in a patient-specific fashion prior to surgery. To that end, a robotic replica of a person's spine was 3D printed, modified to include an artificial disc implant, and outfitted with a soft magnetic sensor array. The aims of this study are threefold: first, to evaluate the potential of a soft magnetic sensor array to detect the location and amplitude of applied loads; second, to use the soft magnetic sensor array in a 3D printed human spine replica to distinguish between five different robotically actuated postures; and third, to compare the efficacy of four different machine learning algorithms to classify the loads, amplitudes, and postures obtained from the first and second aims. Benchtop experiments showed that the soft magnetic sensor array was capable of precisely detecting the location and amplitude of forces, which were successfully classified by four different machine learning algorithms that were compared for their capabilities: Support Vector Machine (SVM), K-Nearest Neighbor (KNN), Random Forest (RF), and Artificial Neural Network (ANN). In particular, the RF and ANN algorithms were able to classify locations of loads applied $3.25 \mathrm{~mm}$ apart with $98.39 \% \pm 1.50 \%$ and $98.05 \% \pm 1.56 \%$ accuracies, respectively. Furthermore, the ANN had an accuracy of $94.46 \% \pm 2.84 \%$ to classify the location that a $10 \mathrm{~g}$ load was applied. The artificial disc-implanted spine replica was subjected to flexion and extension by a robotic arm. Five different postures of the spine were successfully classified with $100 \% \pm 0.0 \%$ accuracy with the ANN using the soft magnetic sensor array. All results indicated that the magnetic sensor array has promising potential to generate data prior to invasive surgeries that could be utilized to preoperatively assess the suitability of a particular intervention for specific patients and to potentially assist the postoperative care of people with cervical disc implants.
\end{abstract}

Keywords: soft magnet; sensor array; machine learning; 3D printing; cervical spine; artificial disc

\section{Introduction}

Cervical spine degenerative disc disease can result in spinal cord injury and nerve root compression, leading to painful symptoms such as myelopathy and radiculopathy [1]. A cervical artificial disc implant is a conventional surgical treatment for these patients; however, the mechanical failure of these devices remains a significant complication, especially in patients with multilevel pathology [2,3]. Static disc pressure has been measured using a very small needle-type pressure sensor, but there are few methods to measure intervertebral 
pressure distribution [4,5]. As a result, the surgeon is forced to determine candidacy for cervical disc implants primarily on the findings of diagnostic imaging studies.

As an alternative, the use of wearable sensors in the acquisition of spinal motion parameters has shown promise throughout the literature [6,7]. Their quick and effortless integration makes them a promising technology that may become a supplement to highcost imaging systems [8-10]. Accurate representation of the geometry and kinematics of a patient's spine is the beginning of realistic phantoms and models [11,12]. Measurements of intradiscal pressure could be gained as well in the testing of the physical models. Literature reports several methodologies for creating sensor arrays, which can measure the pressure at several points across a surface [13,14]. However, flexible sensing technologies are required to integrate them into a spine model without inhibiting mobility.

Flexible magnetoelectronics have attracted many researchers in the past few years with different types of soft, compliant magnets [15]. A flexible magnetic sensor array is a new method to realize soft and stretchable magnets by mixing silicone with magnetic powder [16]. These sensors are low-cost, highly sensitive, and easily integrated into robotic systems as the soft medium can be manipulated in many shapes and sizes [16,17]. Recently, Hall effect sensors have been used in conjunction with a wearable magnetic skin for contact location and force measurement [15-17].

The overall goal of this paper was to develop a new approach to enable surgeons to preview the post-operative effects of an artificial disc implant in a patient-specific fashion prior to surgery (Figure 1).

a

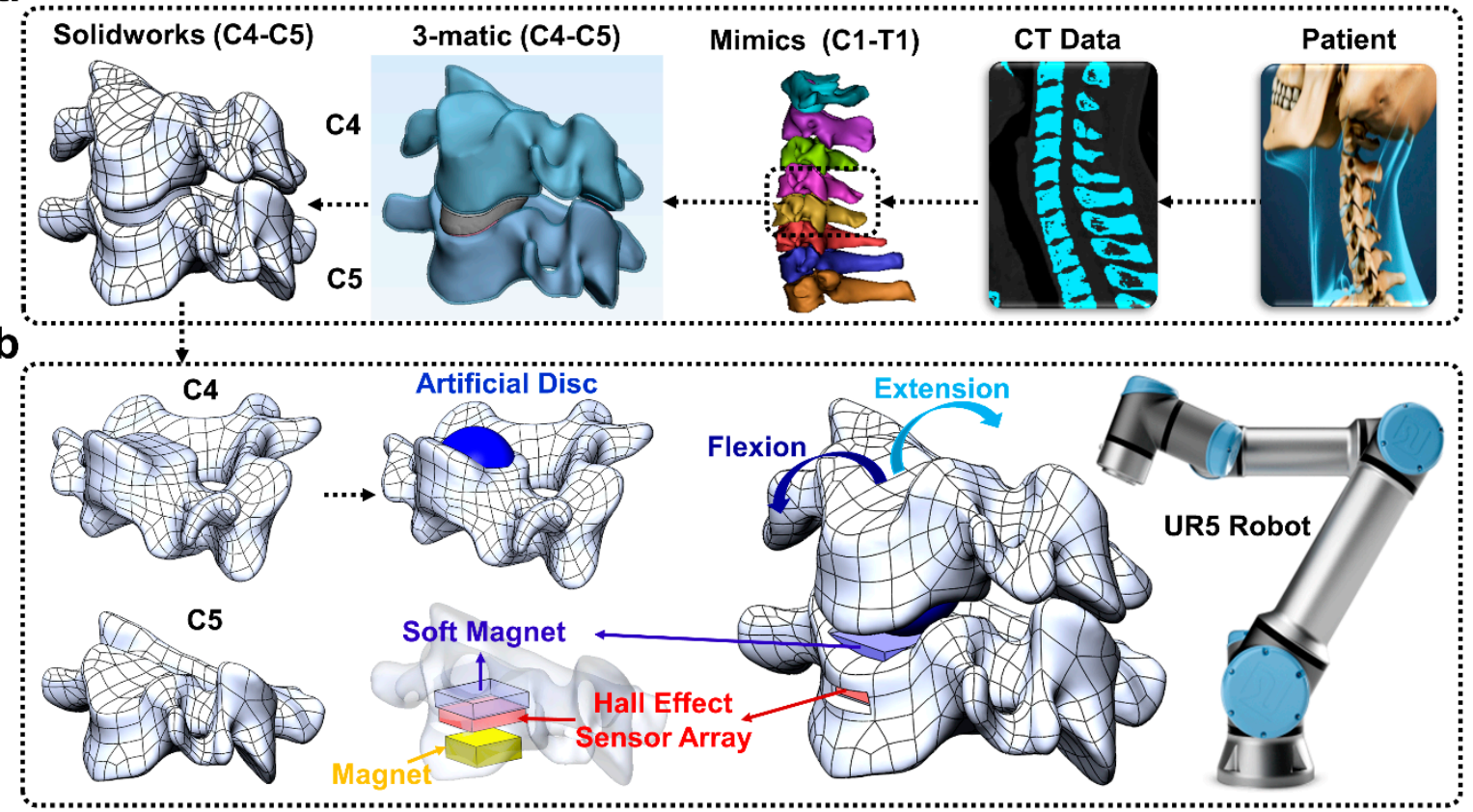

Figure 1. (a) Patient-specific robotic spine model based on a CT scan of the spine. (b) A modified artificial disc was 'implanted' into the C4 cervical spine replica. The soft magnet, Hall effect sensor array board, permanent magnet, and Ecoflex were embedded in the C5 vertebra replica. A robotic arm flexed and extended the cervical spine replica while the intervertebral loads were monitored with the soft magnetic sensor array to classify the spine posture with four different machine learning algorithms.

\section{Materials and Methods}

The soft magnet was fabricated and placed atop a $3 \times 3$ array of Hall effect sensors. The soft sensing magnet comprised magnetic ferrofluid (Apex) mixed with a soft, stretchable elastomer (Figure 2(ai)). Deformation of the soft magnet by external loads displaced the magnetic particles within the soft magnet, and the resulting changes in the magnetic field 
were detected by the Hall effect sensor array (Figure 2(aii,aiii)). Four machine learning algorithms were compared to classify the amplitude and the locations that external loads were applied based on the sensor values reported by the nine Hall effect sensors on the $3 \times 3$ grid during robotic probing experiments (Figure $2 b$ ). The soft magnetic sensor array was next integrated into the artificial disc-implanted robotic replica of the human spine (Figure 1a) that was flexed and extended by a robotic arm (Figure 1b). Four different machine learning algorithms were next compared for their capabilities to classify five different postures of the human spine robotic replica.

a

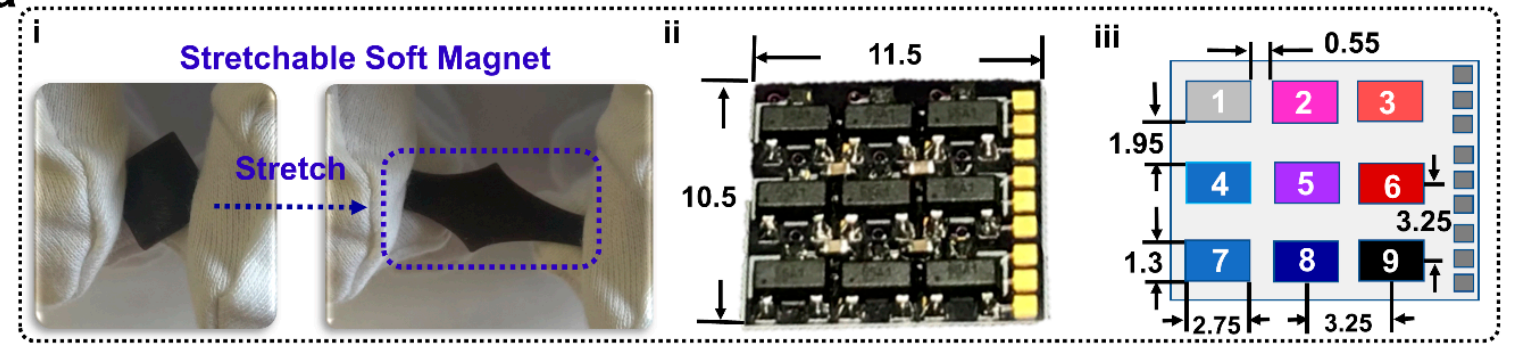

b

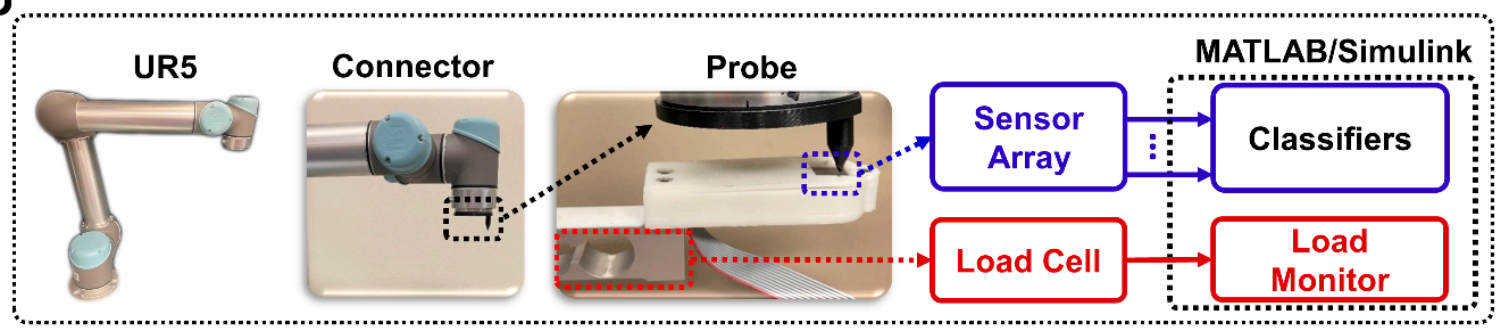

Figure 2. Robotically probing soft magnet to classify location and amplitude of external loads. (ai) The stretchable soft magnet. (aii) The Hall effect sensor array PCB with dimensional units of $\mathrm{mm}$. (aiii) Each individual Hall effect sensor taxel position was numbered and physical dimensions were labeled with units of $\mathrm{mm}$. (b) The UR5 applied loads to the soft magnet that was placed atop the $3 \times 3$ Hall effect sensor array within the 3 D-printed housing. These nine Hall effect sensor signals were recorded in Simulink and classified with four machine learning algorithms in MATLAB.

\subsection{Soft Magnet Fabrication}

The soft magnet was realized by mixing Ecoflex 00-50 (Smooth-On) and ferrofluid magnetic liquid. Two variations of the soft magnetic sensor array were developed and compared by altering the ratios of Ecoflex and ferrofluid with the following weight ratios (Ecoflex part A-Ecoflex part B-ferrofluid): 1:1:0.2 (10\% ferrofluid), 1:1:0.3 (15\% ferrofluid). These ratios were mixed, degassed, poured into square $3 \mathrm{D}$ printed molds, covered with the mold cap, and allowed to cure for at least $12 \mathrm{~h}$.

\subsection{Robotically Probing Soft Magnets to Classify Location and Amplitude}

The soft magnet, Hall effect sensor array, and permanent magnet were assembled within a 3D printed sensor housing (Figure 2a) and screwed securely to the end of a $2 \mathrm{~kg}$ load cell (LSP-2, Transducer Techniques, Temecula, CA, USA) that was clamped onto a desktop (Figure 2b). A $10 \mathrm{~mm} \times 10 \mathrm{~mm}$ square permanent magnet was located $3 \mathrm{~mm}$ below the Hall effect sensor array PCB to strengthen the impact of displacing the magnetic particles within the soft magnet near the Hall effect sensors. The Hall effect sensor array PCB was designed in Eagle and fabricated by OSH Park (Lake Oswego, OR, USA). Nine Hall effect sensors (DRV5055A1QDBZR with the A1 model corresponding to the sensitivity option of $100 \mathrm{mV} / \mathrm{mT}$ and a sensing range of $\pm 21 \mathrm{mT}$; Texas Instruments, Dallas, $\mathrm{TX}$, USA) were next soldered to the PCB (Figure 2(aii)). The Hall effect sensor array and the load cell were interfaced with a computer via a 16-bit PCIe-6323 data acquisition (DAQ) card (National Instruments, Austin, TX, USA). Data were sampled with a $\pm 10 \mathrm{~V}$ range 
producing a resolution of $305 \mu \mathrm{V}$. To amplify the response of the load cell, an INA131 (Texas Instruments) instrumentation amplifier was used.

A UR5 robotic arm (Universal Robots, Odense, Denmark) was bolted onto a metal table adjacent to the soft magnetic sensor array. A probe was 3D printed using Ultimaker 3 (Ultimaker, Zaltbommel, the Netherlands) and bolted to the end effector of the UR5 arm (Figure $2 \mathrm{~b}$ ). The probe had a flat tip with a $4 \mathrm{~mm}^{2}$ surface area. Simulink (The MathWorks, Natick, MA, USA) was used as the data collection software for both the load cell and the Hall effect sensor array. Data were low pass filtered and sampled at $1 \mathrm{kHz}$.

The UR5 was programmed using Universal Robot's proprietary user interface, PolyScope. This interface allowed programming the robot through manual control of the robotic arm to set waypoints, directing its movement through a sequence of target positions and loads, as measured by the load cell. The robot was programmed to probe the sensor in 9 different locations in a $3 \times 3$ grid; each probe location was directly above a different Hall effect sensor (Figure 2(aiii)). Thirty repetitions at each of the nine taxels (tactile pixel) were done with six different loads: $5 \mathrm{~g}, 10 \mathrm{~g}, 20 \mathrm{~g}, 50 \mathrm{~g}, 75 \mathrm{~g}$, and $100 \mathrm{~g}$. This procedure was replicated for both soft magnets that were comprised of $10 \%$ or $15 \%$ ferrofluid. This produced 3240 datasets ( 9 locations $\times 30$ repetitions $\times 6$ loads $\times 2$ soft magnets) that were used to train four different machine learning algorithms which were compared for load amplitude and location detection accuracy with each soft magnet. The correlation between load and Hall effect sensor readings was also plotted with a linear fit model at each taxel location in MATLAB. From this, the sensitivity in $\mathrm{mV} / \mathrm{g}$ was reported along with the $\mathrm{R}^{2}$ values to quantify the goodness of fit of the linear models.

To evaluate the measurement uncertainty of the soft magnetic sensors, we followed the guidelines from the National Institute of Standards and Technology (NIST) [18]. In our application, the main sources of measurement uncertainty in the force sensing application were caused by deviations of the measured force from the value predicted by the linear models for the external LSP-2 load cell $\left(U_{\text {Load Cell }}\right)$ and also each taxel on the soft magnetic sensor array $\left(U_{\text {Taxel }, i}\right)$. To calculate the uncertainty $(U)$ for both of these sources, we used the same procedure outlined by NIST based on the difference between the measured values and the values predicted by the models fit to the data:

$$
U^{2}=\frac{\sum_{j=1}^{n} d_{j}^{2}}{(n-m)}
$$

where $d_{j}$ is the difference between the measured response and the calculated response using the linear models fit to the data, $n$ is the number of measurement repetitions and $\mathrm{m}$ is the order of the polynomial modeling the data plus one $(m=2)$. We fit linear models to data from the load cell and each taxel on the sensor array for both soft magnets. The number of repetitions for each of the nine taxels and the load cell was $n=30$. We next calculated the overall measurement uncertainty for each taxel $i$ of the sensor array:

$$
U_{i}{ }^{2}=U_{\text {Taxel }, i^{2}}+U_{\text {Load Cell }}{ }^{2}
$$

$U_{\text {Taxel, } i}$ is the measurement uncertainty of each taxel $i$ on the sensor array and $U_{\text {Load Cell }}$ is the uncertainty of the load cell, both of which were calculated using (1). $U_{i}$ is the overall measurement uncertainty for each taxel $i$ (Figure 2(aiii)), including the load cell measurement uncertainty.

\subsection{Robotic Replica of Human Spine to Preview Artificial Disc Implantation}

The CT scan of a person's cervical spine was imported into Solidworks ${ }^{\mathrm{TM}}$ (Waltham, MA, USA) to produce a CAD model (C4-C5 cervical spine, Figure 1a) using Mimics/3matic $[19,20]$ and Hypermesh [21-23]. The C4 vertebra model was virtually "implanted" with an artificial disc (modified from the ProDisc-C [24]) to preview the effects of the intervention prior to surgery (Figure $1 \mathrm{~b}$ ). The $\mathrm{C} 4$ vertebra model was also altered to allow a mechanical connection to the UR5 robotic arm end effector. This concept has a 
powerful potential to enable surgeons to preview and compare the effects of different surgical interventions in a patient-specific manner using robotically actuated spine replica. Similarly, the C5 vertebra was modified to house the soft magnet, Hall effect sensor array board, and permanent magnet. The C5 vertebra replica was also designed so that it could be bolted to an LSP-35 load cell (Transducer Techniques, Temecula, CA, USA) for load monitoring (Figure $1 \mathrm{~b}$ ). The $\mathrm{C} 4$ and $\mathrm{C} 5$ vertebrae were subsequently 3D printed with Ultimaker 3.

The artificial disc 'implanted' C4 replica was bolted to the UR5 arm, which was programmed to flex and extend the $\mathrm{C} 4$ vertebra relative to the $\mathrm{C} 5$ vertebra (30 times for each soft magnet). The soft magnetic sensor array was used to classify five different postures of the spine replica: center, mid-flexion, flexion, mid-extension, extension.

\subsection{Machine Learning Classification Approach}

Extracting features from time-domain data is helpful to train a machine learning classifier to recognize the patterns hidden within the data. In some cases, frequency features are extracted to help classify patterns within the data [25]. Another way to produce features is through statistical information to represent the data. In this paper, 15 statistical features were extracted from each dataset (Table 1). After extracting the statistical features from the time domain data, the feature matrices were used to train and compare four distinct machine learning algorithms: Support Vector Machine (SVM), K-Nearest Neighbor (KNN), Random Forest (RF), and Artificial Neural Network (ANN).

Table 1. Statistical Features Extracted for Training Machine Learning Algorithms.

\begin{tabular}{lc}
\hline & Statistical Features for Algorithm Training \\
\hline 1 & Mean \\
\hline 3 & Maximum Element of a Vector \\
\hline 4 & Minimum Element of a Vector \\
\hline 5 & Sum of Vector Elements \\
\hline 6 & Standard Deviation \\
\hline 7 & Range of Values \\
\hline 8 & Root Mean Square \\
\hline 9 & Skewtosis \\
\hline 10 & Peak Magnitude to RMS ratio \\
\hline 11 & Most Frequent Values in an Array \\
\hline 12 & Variance \\
\hline 13 & Interquartile Range \\
\hline 14 & Mean Absolute Deviation \\
\hline 15 &
\end{tabular}

The SVM algorithm creates a separating hyperplane in the feature space between all the classes [26]. The SVM algorithm uses the extracted features for the training portion to generate the hyperplane. The maximum separation margin between the classes is calculated and the hyperplane is constructed in the middle of the margin. The KNN algorithm calculates the shortest distance between a query and classifies the new samples based on the majority vote of its neighbors. The classes are assigned based on the most common vote amongst its $\mathrm{K}$ nearest neighbor measured by the shortest distance. The points in the extracted features select the specified $\mathrm{K}$ number closest to the query to vote for the most repeated class number [27]. The RF algorithm contains a collection of tree predictors such that each tree is initialized with random values independently from each 
other with the same distribution of all trees in the forest [28]. As the number of trees in the forest increase, the generalization error approaches the minimum limit to ensure the best performance in classification and prediction. The RF is a very effective machine learning tool since it contains multiple trees-each tree is an independent classifier, and its classification accuracy is independent of other trees in the forest. The RF algorithm used in this study was designed with 500 trees to perform the classification assignment. In general, a forest with more trees produces more robust predictions which lead to higher classification accuracy. The neural network toolbox in MATLAB (nprtool) was used to generate a feedforward network to train and test the ANN [29]. A three-layer feedforward network with 100 hidden neurons with a sigmoid activation function for the hidden layer and output neurons with a softmax activation function for the output layer was used to classify the collected data. The extracted statistical features were fed into the ANN through the input layer which consists of the input neurons. The neurons in the output layer represent the output classes. The performance of the network was evaluated using cross-entropy and confusion matrices, and the network was trained with scaled conjugate gradient backpropagation.

In the robotic probing experiments, the collected data were used to train and test the machine learning classifiers in two different configurations. In the first configuration, the machine learning classifiers were used to distinguish between 10 different classes ( 9 taxel locations and no-touch), and in the second configuration, the machine learning classifiers were used to distinguish between 6 different loads ( $5 \mathrm{~g}, 10 \mathrm{~g}, 20 \mathrm{~g}, 50 \mathrm{~g}, 75 \mathrm{~g}, 100 \mathrm{~g}$ ). To evaluate the sensor for the artificial disc-implanted spine replica application (Figure 1b), five output classes were defined for each classifier corresponding to five different postures of the spine that were robotically actuated: center, mid-flexion, flexion, mid-extension, and extension.

To train and test the ANN, the feature extracted dataset was separated into 3 groups: $70 \%$ training dataset, $15 \%$ validation dataset, and $15 \%$ testing dataset. The ANN was trained, and the network parameters were modified according to the error generated with the training dataset. The validation dataset was used to measure network generalization error and to stop training when the generalization error stopped improving, while the testing dataset was used to provide an independent evaluation of network performance after the training, and it is independent of the validation dataset. The training process automatically stopped when generalization error stopped improving, as indicated by an increase in the cross-entropy error of the validation dataset. For the SVM, KNN, and RF machine learning algorithms, the extracted features were subdivided randomly into two categories, the training data which included $80 \%$ of the feature data, and the testing data which included the remaining $20 \%$ of the extracted feature dataset. Overfitting can cause the generalized performance of any classification model to decrease significantly. To avoid overfitting problems, feature data were swapped randomly before training and testing the classifier models. To avoid any biases, each classifier model was run 10 times with randomized selection of the $80 \%$ training features and $20 \%$ testing features and the average and standard deviation of the classification accuracy was reported for each of the four algorithms.

\subsection{Statistical Analysis}

Two different three-factor analysis of variance (ANOVA) tests were performed to assess the statistical significance of the results from the two different configurations of machine learning algorithms used with the robotic probing experiments. In each case, the dependent variable was the classification accuracy of the machine learning algorithms. The independent variables for the first configuration were the taxel location where loads were applied (9 taxels), the soft magnet composition (10\% or $15 \%$ ferrofluid), and the machine learning algorithm (ANN, KNN, SVM, or RF). The independent variables for the second configuration were the load amplitude (6 loads), the soft magnetic skin composition (10\% or $15 \%$ ferrofluid), and the machine learning algorithm (ANN, KNN, SVM or RF). 
A two-factor ANOVA was performed on the robotically actuated spine replica experiments. The independent variables were the soft magnet composition (10\% or $15 \%$ ferrofluid) and the four different machine learning algorithms. The dependent variable was the accuracy to classify the five different postures of the spine. A $p$-value $<0.01$ was used in all cases for statistical significance.

\section{Results}

Sample time-domain data from the soft magnetic sensor array with the $10 \%$ ferrofluid composition showed the taxels' responses as a $75 \mathrm{~g}$ load was applied repeatedly in nine different locations (Figure 3a). Spatially organized responses of each taxel showed similar trends of signals responding to different probing locations with the $100 \mathrm{~g}$ load (Figure 3(bii)). Corresponding time response data with a $75 \mathrm{~g}$ load (Figure 4a) and spatially organized responses with the $100 \mathrm{~g}$ load (Figure 4(bii)) show similar trends with the $15 \%$ ferrofluid soft magnet composition. The correlation between load and voltage measured by each of the nine Hall effect sensors for both soft magnets is reported in Figure 3(bi) and Figure 4(bi). A linear model was fit to these data. Averaged across all nine taxels, the $\mathrm{R}^{2}$ value of the linear fit for the $10 \%$ ferrofluid soft magnet was $0.88 \pm 0.03$ and the $\mathrm{R}^{2}$ value for the $15 \%$ ferrofluid soft magnet was $0.90 \pm 0.04$ (Table 2). Averaged across all nine taxels, the sensitivity for the $10 \%$ ferrofluid soft magnet was $0.09 \mathrm{mV} / \mathrm{g} \pm 0.02 \mathrm{mV} / \mathrm{g}$ (Figure 3(bi), Table 2). The mean sensitivity of the $15 \%$ ferrofluid soft magnet was $0.14 \mathrm{mV} / \mathrm{g} \pm 0.03 \mathrm{mV} / \mathrm{g}$ (Figure 4(bi), Table 2). The measurement uncertainties for the 9 taxels that were calculated according to (2) ranged between $3.23 \mathrm{~g}$ and $6.24 \mathrm{~g}$ for the 15\% ferrofluid Soft Magnet (Table 3). Uncertainties for the $10 \%$ ferrofluid soft magnet followed similar trends with moderately higher levels of measurement uncertainty.

Table 2. Linear fit model parameters for both soft magnets. The linear model polynomials have a form: Hall effect sensor $(\mathrm{mV})=\mathrm{P} 1 *(\operatorname{Load}(\mathrm{g}))+\mathrm{P} 2$.

\begin{tabular}{ccccccc}
\hline & \multicolumn{2}{c}{ Soft Magnet 1 (10\% Ferrofluid) } & \multicolumn{2}{c}{ Soft Magnet 2 (15\% Ferrofluid) } \\
\hline Taxel & $\mathbf{P 1}(\mathbf{m V} / \mathbf{g})$ & $\mathbf{P 2} \mathbf{( m V )}$ & $\mathbf{R}^{\mathbf{2}}$ & $\mathbf{P 1} \mathbf{( \mathbf { m V } / \mathbf { g } )}$ & $\mathbf{P 2} \mathbf{( \mathbf { m V } )}$ & $\mathbf{R}^{\mathbf{2}}$ \\
\hline 1 & 0.09 & 0.15 & 0.85 & 0.15 & 0.02 & 0.91 \\
\hline 2 & 0.11 & -0.92 & 0.91 & 0.17 & 0.31 & 0.92 \\
\hline 3 & 0.07 & 0.18 & 0.87 & 0.12 & -0.16 & 0.87 \\
\hline 4 & 0.11 & -1.45 & 0.92 & 0.18 & 0.86 & 0.94 \\
\hline 5 & 0.11 & -1.36 & 0.89 & 0.15 & -3.12 & 0.91 \\
\hline 6 & 0.07 & -1.83 & 0.87 & 0.13 & 0.24 & 0.91 \\
\hline 7 & 0.09 & -1.66 & 0.90 & 0.11 & -0.86 & 0.88 \\
\hline 9 & 0.08 & -1.03 & 0.86 & 0.16 & 0.02 & 0.93 \\
\hline Mean & 0.05 & -0.74 & 0.81 & 0.08 & -0.18 & 0.83 \\
\hline $\begin{array}{c}\text { Standard } \\
\text { Deviation }\end{array}$ & 0.02 & -0.96 & 0.88 & 0.14 & -0.32 & 0.90 \\
\hline
\end{tabular}




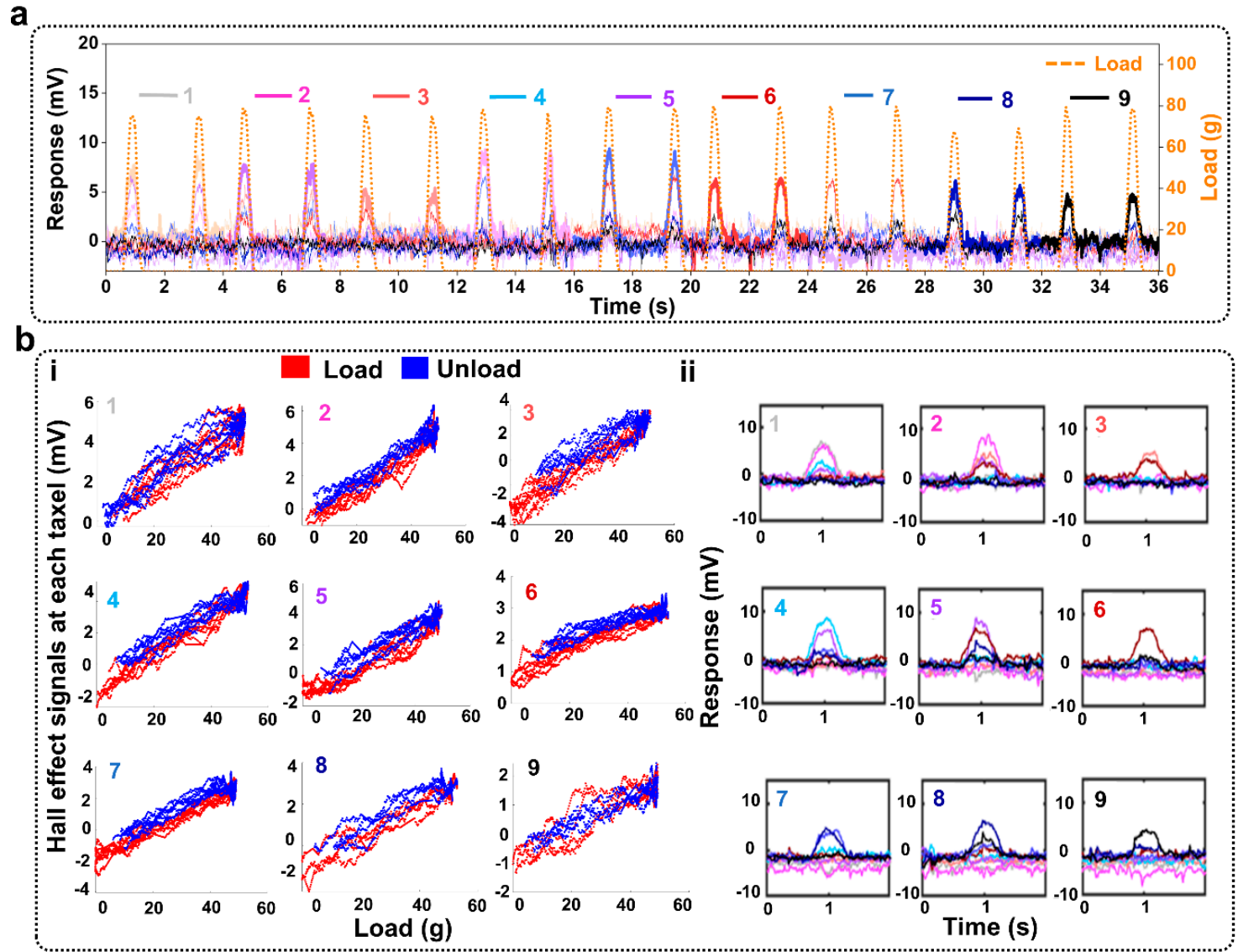

C

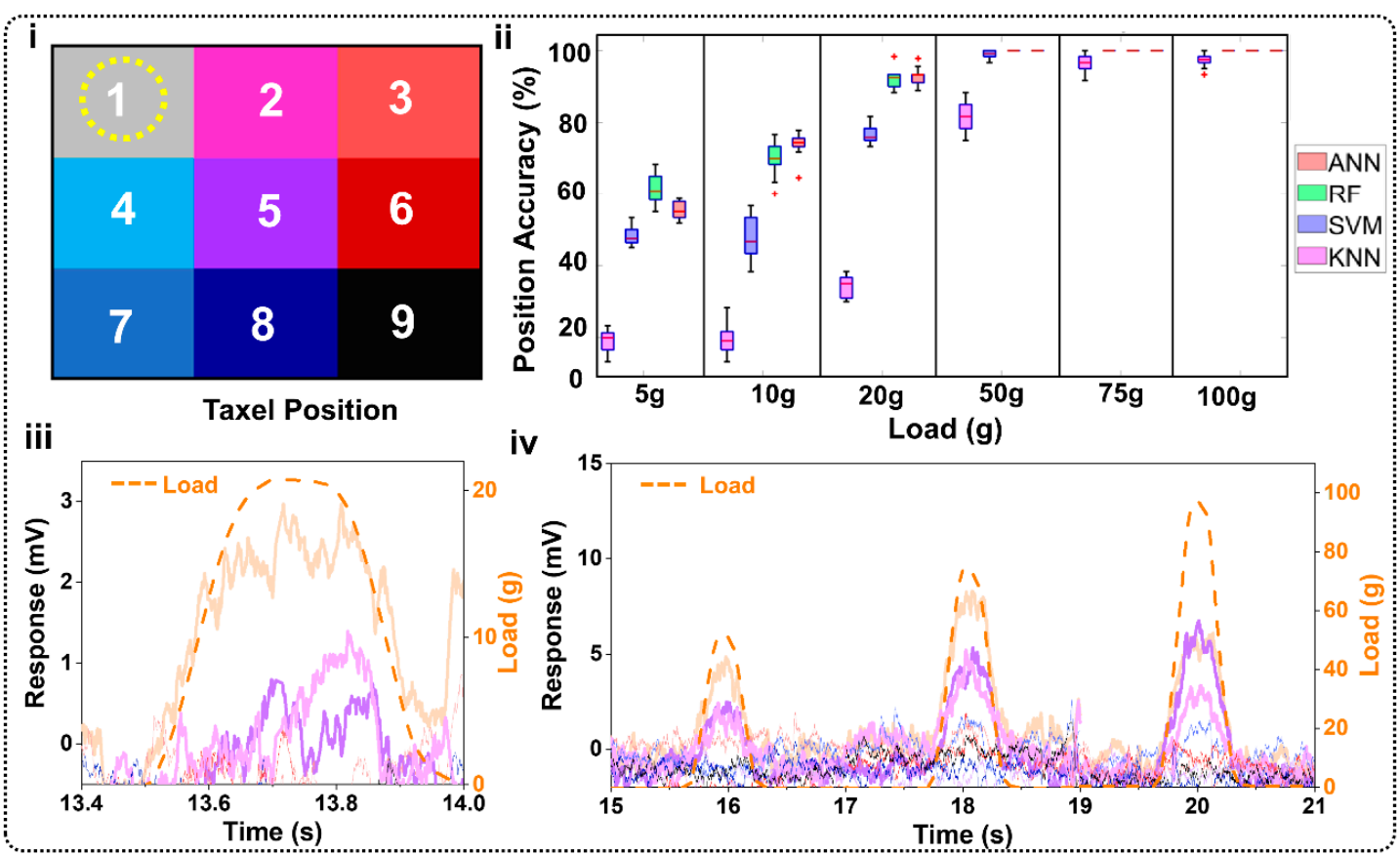

Figure 3. Classification accuracy of $10 \%$ ferrofluid soft magnetic sensor array. (a) Illustrative responses and load cell data at the nine taxel locations with a $75 \mathrm{~g}$ load. (bi) Load changes with Hall effect signals at each taxel, (bii) Individual taxel responses under $100 \mathrm{~g}$ load. (ci) Hall effect sensor taxel position. (cii) Classification accuracies to detect the different locations a load was applied with each of the six different loads ( $n=30$ repetitions/load). (ciii) Taxel responses under $20 \mathrm{~g}$ load applied at taxel 1. (civ) All taxel responses under different loads (50 g, 75 g, $100 \mathrm{~g}$ ) applied at taxel 1. 
a

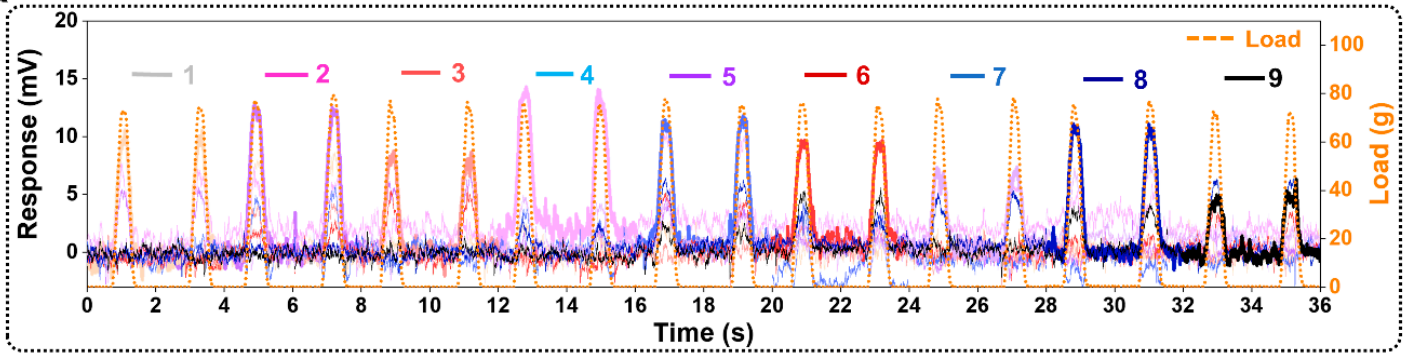

b
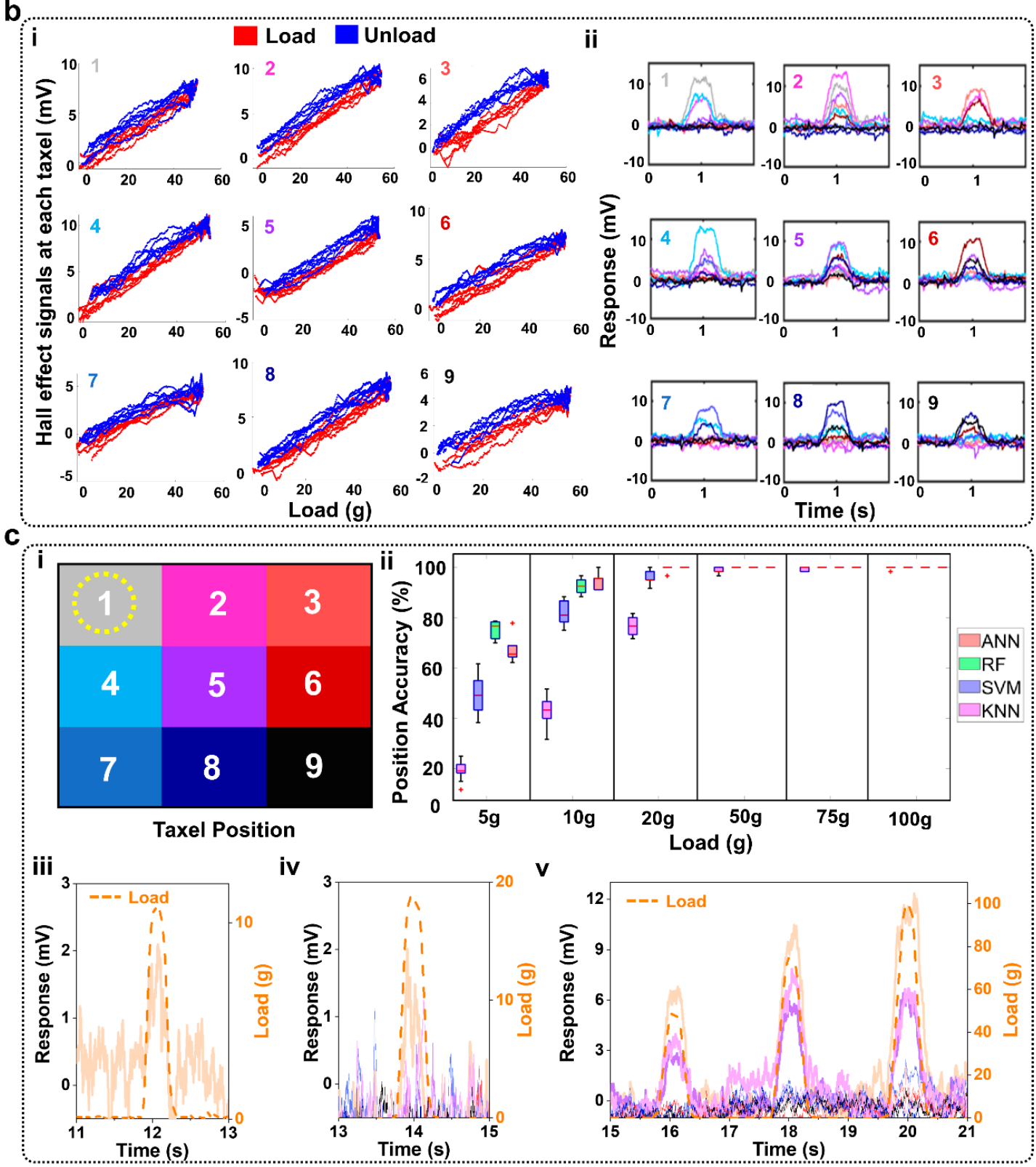

Figure 4. Classification accuracy of $15 \%$ ferrofluid Hall effect sensor array. (a) Illustrative responses and load data at the nine taxel locations. (bi) Load changes with Hall effect signals at each taxel, (bii) Individual taxel responses under $100 \mathrm{~g}$ load. (ci) Taxel location color code indicating illustrative data is from taxel 1. (cii) Classification accuracies to detect the different locations a load was applied with each of the six different loads ( $n=30$ repetitions $/$ load). (ciii) Taxel 1 response to the $10 \mathrm{~g}$ load applied at taxel 1. (civ) All taxel responses from a $20 \mathrm{~g}$ load applied at taxel 1. (cv) All taxel responses under different loads (50 g, $75 \mathrm{~g}, 100 \mathrm{~g}$ ) applied at taxel 1. 
Table 3. Sensor uncertainties (2) for each taxel i from both soft magnets.

\begin{tabular}{ccc}
\hline & Soft Magnet 1 (10\% Ferrofluid) & Soft Magnet 2 (15\% Ferrofluid) \\
\hline Taxel & Measurement Uncertainty (g) & Measurement Uncertainty (g) \\
\hline 1 & 6.14 & 5.21 \\
\hline 2 & 5.21 & 3.81 \\
\hline 3 & 8.16 & 5.55 \\
\hline 4 & 6.87 & 6.24 \\
\hline 5 & 4.88 & 4.84 \\
\hline 6 & 7.15 & 3.23 \\
\hline 7 & 7.82 & 4.39 \\
\hline 8 & 6.35 & 3.34 \\
\hline 9 & 11.49 & 5.71 \\
\hline
\end{tabular}

\subsection{Classifying Locations of Applied Loads}

At each location, the applied load triggered distinct patterns of taxel responses that were classified by the four different machine learning algorithms to distinguish between the probing locations for given loads. These algorithms showed high classification accuracies with both the 10\% ferrofluid (Figure 3(cii)) and 15\% ferrofluid soft magnets (Figure 4(cii)). In particular, the RF and ANN accuracies were respectively 98.39\% $\pm 1.50 \%$ and $98.05 \% \pm 1.56 \%$ with the $15 \%$ ferrofluid soft magnet (Figure 4 (cii)).

Three-factor ANOVA showed that the classification accuracies were significantly impacted by the four different algorithms $(p<0.01)$, the two soft magnets $(p<0.01)$, and the nine different taxel locations $(p<0.01)$. The interaction between the classification algorithms and the load amplitudes was significant $(p<0.01)$. One illustration of this interaction can be seen with the higher accuracy of the RF compared to the ANN at the $5 \mathrm{~g}$ load whereas the ANN had a higher accuracy than the RF with the $10 \mathrm{~g}$ load. The interaction between the classification algorithms and the two different soft magnets was also significant $(p<0.01)$. This interaction can be seen, for example, with the accuracy of the KNN with $10 \%$ ferrofluid soft magnet (Figure 3(cii)) being significantly lower than the RF with 15\% ferrofluid soft magnet (Figure 4(cii)). The load amplitudes and the ferrofluid percentage of the soft magnets also significantly interacted $(p<0.01)$. This interaction can be seen, in one illustrative case, as the accuracies for the $5 \mathrm{~g}$ loads with the $10 \%$ ferrofluid soft magnet (Figure 3(cii)) were significantly lower than the classification accuracies with the $10 \mathrm{~g}$ load and the $15 \%$ ferrofluid soft magnet (Figure 4(cii)). The three-factor interaction is significantly different $(p<0.01)$ because of the many cases where the classification accuracies were significantly different, for example, the SVM with $5 \mathrm{~g}$ load and the $10 \%$ ferrofluid soft magnet compared to the RF with the $10 \mathrm{~g}$ and $15 \%$ ferrofluid soft magnet.

\subsection{Distinguishing between Different Loads at Each Taxel Location}

The machine learning algorithms were able to distinguish between all the loads applied to each location with high classification accuracy for loads $\geq 20 \mathrm{~g}$ with the $10 \%$ ferrofluid soft magnet (Figure S1a) and loads $\geq 10 \mathrm{~g}$ with the $15 \%$ ferrofluid soft magnet (Figure S1b, Table S1). Generally, the classification accuracies were higher when the sensor was manufactured with $15 \%$ ferrofluid than when the sensor was manufactured with $10 \%$ ferrofluid. The highest accuracies were $98.39 \% \pm 0.93 \%$ with the RF and $98.04 \% \pm 1.37 \%$ with the ANN when the sensor was manufactured with 15\% ferrofluid, while the highest accuracy was $96.18 \% \pm 1.41 \%$ for the ANN when the sensor was manufactured with $10 \%$ ferrofluid (Table S1).

When detecting different loads at a given taxel, the three-factor ANOVA indicated that the classification accuracies were significantly impacted by the taxel locations, soft magnets ( $10 \%$ ferrofluid, and $15 \%$ ferrofluid), and different algorithms $(p<0.01)$. The inter- 
actions between the machine learning algorithms and the taxel locations were significant $(p<0.01)$. One case illustrating this interaction is that the RF accuracy was significantly lower than the ANN accuracy at taxel 4 while the RF accuracy was significantly higher than the ANN accuracy at taxel 7 with the $10 \%$ ferrofluid soft magnet (Figure S1a). The interaction between locations and different ferrofluid percentage is significant $(p<0.01)$ due to the different cases like taxel 5 with 10\% ferrofluid (Figure S1a) in comparison to taxel 4 with 15\% ferrofluid (Figure S1b). The interaction between the four machine learning algorithms with the two different soft magnets was significant also $(p<0.01)$ due to the mean response for KNN in 10\% ferrofluid being significantly different than RF in 15\% ferrofluid, for example (Table S1). The three-factor interaction among load amplitude, soft magnet, and classification algorithm were also significant $(p<0.01)$ because of the significantly different cases such as the RF with $5 \mathrm{~g}$ load and the $10 \%$ ferrofluid soft magnet compared to the ANN with the $10 \mathrm{~g}$ and $15 \%$ ferrofluid soft magnet.

\subsection{Intervertebral Monitoring of Human Spine Robotic Replica for Posture Classification}

Five spine postures (center, mid-flexion, flexion, mid-extension, and extension) were classified by the four machine learning algorithms with both the $10 \%$ and $15 \%$ ferrofluid soft magnetic sensor arrays. When the UR5 arm robotically flexed and extended the human spine robotic replica with the artificial disc implant (Figure 5a), the sensor signals increased or decreased significantly with both the $10 \%$ ferrofluid (Figure $5 \mathrm{~b}$ ) and $15 \%$ ferrofluid soft magnetic sensor arrays (Figure 5c). The ANN performed the best with the $10 \%$ ferrofluid soft magnetic sensor array with a $100 \% \pm 0.0 \%$ success rate (Table 4 ), while both $\mathrm{KNN}$ and ANN performed exceptionally with the 15\% magnetic sensor array with $99.66 \% \pm 1.05 \%$ and $99.14 \% \pm 1.81 \%$ accuracies, respectively (Table 4 ).

a
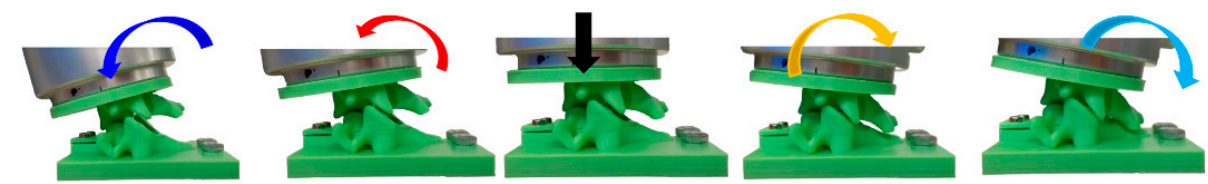

b

Flexion

Mid-Flexion

Center

Mid-Extension

Extension

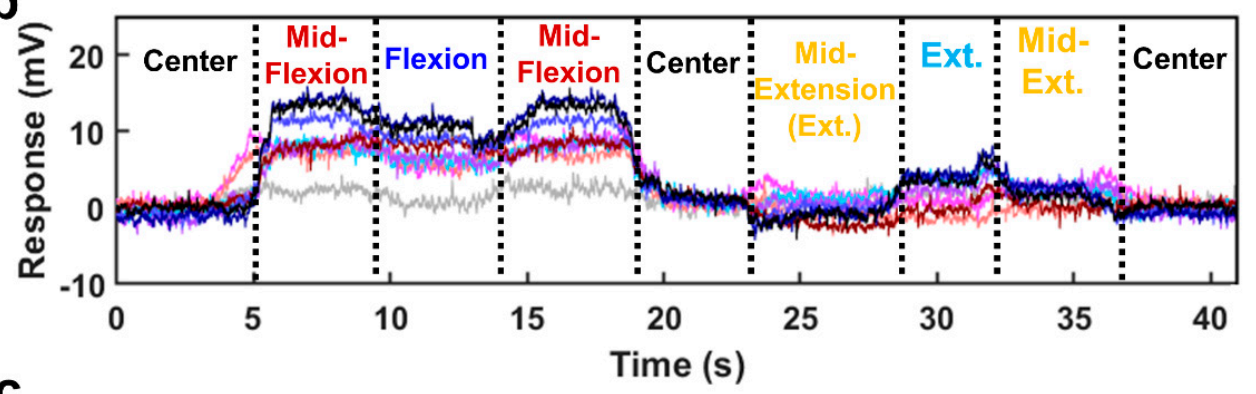

C

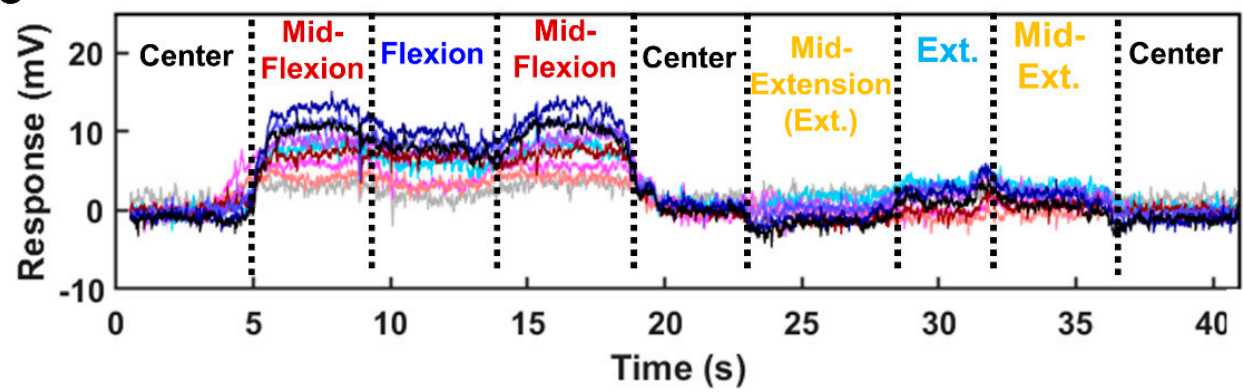

Figure 5. Human spine robotic replica posture measurement via intervertebral monitoring. (a) Five robotically actuated postures of the artificial disc-implanted spine replica were classified by four machine learning algorithms during flexion and extension motions. (b) Taxel responses measured with the $10 \%$ ferrofluid soft magnet. (c) Taxel responses measured with the $15 \%$ ferrofluid soft magnet. 
Table 4. Machine learning algorithm accuracies to detect the five different postures of the spine replica with the $10 \%$ ferrofluid and $15 \%$ ferrofluid soft magnets.

\begin{tabular}{|c|c|c|}
\hline Classification Algorithms & $\begin{array}{c}\text { Accuracy } \\
\text { (10\% Ferrofluid) }\end{array}$ & $\begin{array}{c}\text { Accuracy } \\
\text { (15\% Ferrofluid) }\end{array}$ \\
\hline K-Nearest Neighbors (KNN) & $94.00 \% \pm 3.78 \%$ & $99.66 \% \pm 1.05 \%$ \\
\hline Support Vector Machine (SVM) & $97.33 \% \pm 3.06 \%$ & $99.00 \% \pm 1.60 \%$ \\
\hline Random Forest (RF) & $99.33 \% \pm 1.40 \%$ & $98.33 \% \pm 1.75 \%$ \\
\hline Artificial Neural Network (ANN) & $100.00 \% \pm 0.00 \%$ & $99.14 \% \pm 1.81 \%$ \\
\hline
\end{tabular}

Two-factor ANOVA indicated that classification accuracies were significantly impacted $(p<0.01)$ by both the machine learning algorithms and the soft magnets. The interaction between the classification algorithms and the two soft magnets was also significant $(p<0.01)$. One example of this interaction can be seen from the mean response of the KNN, which was lower than the RF with the $10 \%$ ferrofluid soft magnet (Table 4 ), whereas the KNN was more accurate than the RF with the $15 \%$ ferrofluid soft magnet (Table 4).

\section{Discussion}

A new soft magnetic sensor array system was successfully fabricated to achieve intervertebral load monitoring of robotically actuated human spine replica, promising the potential to preview artificial disc implant suitability in a patient-specific fashion. Results showed that the soft magnetic sensor array system has the high capability to classify five different postures of the spine, which can be a predictor of different problems of the spine that people experience [30]. In comparing the two soft magnets on the algorithms' abilities to detect different loads and locations, the soft magnet with $15 \%$ ferrofluid generally produced higher classification accuracy in detecting the different loads applied to each taxel (Table S1, Figure S1). As the load amplitudes increased, the soft magnets yielded very comparable classification accuracies; however, the main difference between the soft magnets was with the low amplitude loads. The location where the $10 \mathrm{~g}$ load was applied was classified with high accuracy using the ANN and RF algorithms with the $15 \%$ ferrofluid soft magnet (Figure 4(cii,ciii)), which was much more accurate in comparison to the $10 \%$ ferrofluid soft magnet data (Figure 3(cii)). From these findings, it is hypothesized that the sensitivity of the system could be improved by further optimizing the ratio of magnetic particles within the elastic material, which must be counterbalanced against the impact that would have upon the mechanical properties of the soft magnet sensor array. Comparing the algorithms, the ANN had classification accuracy $>99 \%$ with both soft magnets during the flexion-extension experiments with the spine replica (Figure 5). However, there were several cases where the RF slightly outperformed the ANN in the probing experiments to detect the locations where the load was applied (Figures 3(cii) and 4(cii)). This is an important consideration to bear in mind when choosing an algorithm for a particular classification problem such as contact location, load amplitude, or more specifically, to detect the posture of the spine where the ANN performed the best.

Prior work has shown that the loads on the human cervical spine vary from $120 \mathrm{~N}$ to $1200 \mathrm{~N}$ during daily tasks [31]. However, this net load is distributed across the entire surface of the intervertebral disc and surrounding tissues. The intradiscal pressure of $\mathrm{C} 3-\mathrm{C} 4$ human spine cadavers ranged from $200 \mathrm{kPa}$ to $270 \mathrm{kPa}$ during physiologically relevant flexion and extension movements (Figure 3 in [32]). In this paper, we have applied loads ranging from $5 \mathrm{~g}$ to $100 \mathrm{~g}$ over a surface area of $4 \mathrm{~mm}^{2}$ with a flat-tipped probe. The $100 \mathrm{~g}$ load over a $4 \mathrm{~mm}^{2}$ area produces a physiologically relevant pressure of $245.3 \mathrm{kPa}$, which corresponds quite closely to those reported in [32]. We chose the range of loads from $5 \mathrm{~g}$ to $100 \mathrm{~g}$ to span the range from indetectable to detectable loads to uncover the capabilities of each different classification algorithm at every load and location, which was in general different. The nuanced results regarding the interplay between load amplitude and classification 
accuracy of the different algorithms can be helpful in the future to implement $\mathrm{AI}$ in new sensing applications.

The novel use of machine learning algorithms and the soft magnetic sensor array to classify different postures of a patient's artificial disc-implanted robotic spine replica could also be utilized in conjunction with existing techniques to study and predict whether a patient is a candidate for artificial disc replacement or cage-plate fusion. Moreover, the novel system could help in determining whether a constrained, semi-constrained, or unconstrained device could be the best fit [33]. After surgery, the spine replica could assist in estimating whether there is sufficient motion at the operated level and possibly change the rehabilitation program to prevent calcification and subsequent loss of intended motion [34]. Additionally, motion and angulation data could be correlated with a patient's symptoms or complications to better understand them from a biomechanical standpoint. In combination with wearable sensors on the patient's spine, one could also obtain additional data to ameliorate postoperative care and the overall success of the surgery [35]. At the current time, postoperative instructions for patients with spine implants are qualitative (do as much as you can until the pain starts), creating fears in both the patient and the surgeon [36]. Questions regarding how much bending, lifting, and exercising is permissible after a cervical implant operation could be studied and correlated with biomechanical data generated by the sensorized robotic replica with individually tailored postoperative care that could be prescribed to reduce complications [37]. However, one limitation in this study is that there are no ligaments or muscles in the robotic spine replica. In the future, this could be overcome with a more lifelike tissue phantom approach or with an FEA model to understand the effect of ligaments and muscles on the sensor properties [38]. Furthermore, intervertebral simulations from the FEA model of the spine [38] could be merged with empirical measurements from the robotic replica and wearable sensors from the patient after surgeries to provide multiple viewpoints and a unified set of guidelines for post-operative care. This could lower the likelihood of complications, such as artificial disc subsidence that narrows the neuroforamen space, causing cervicalgia and cervical radiculopathy [39]. In the future, this sensor could also potentially be coupled with CT scans to address the issue of spinal malalignment [40-42].

\section{Conclusions}

We have created a novel robotic replica of a human spine to enable surgeons to preview the effects of surgical interventions prior to the operation. The 3D printed spine replica was modified to include an artificial disc and a soft magnetic sensor array. Benchtop experiments showed that the magnetic sensor array was readily capable of detecting the location (with $3.25 \mathrm{~mm}$ spacing) and amplitude of externally applied loads $\geq 10 \mathrm{~g}$ from a robotic arm, as evidenced by high classification accuracies from the four different machine learning algorithms that were compared. When the soft magnetic sensor array was integrated within the human spine robotic replica, the ANN had the highest accuracy of $100 \%$ to classify five different postures of the robotic spine replica. These results indicated that the integration of the soft magnetic sensor array within the artificial disc 'implanted', robotically actuated spine replica has the potential to generate physiologically relevant data before invasive surgeries, which could be used preoperatively to assess the suitability of a particular intervention for specific patients.

Supplementary Materials: The following supporting information can be downloaded at: https: / / www.mdpi.com/article/10.3390/s22010212/s1, Figure S1: Classification accuracies from four algorithms to detect the load magnitude at each of the nine different taxels. Thirty repetitions of six different loads $(5 \mathrm{~g}, 10 \mathrm{~g}, 20 \mathrm{~g}, 50 \mathrm{~g}, 75 \mathrm{~g}, 100 \mathrm{~g}$ ) were applied to every taxel for both soft magnets. a. $10 \%$ ferrofluid soft sensor array. b. $15 \%$ ferrofluid soft sensor array; Table S1: Overall mean and standard deviation of algorithm accuracies for load amplitude classification at each of the nine taxels; Video S1: Stretchable soft magnet, load at each taxel and intervertebral monitoring of human spine replica. 


\begin{abstract}
Author Contributions: Conceptualization, M.L., C.-T.T., F.D.V., and E.D.E.; methodology, M.L., A.T., M.A.A., and E.D.E.; software, M.L., A.T., M.A.A., and E.D.E.; validation, M.L., and M.A.A.; formal analysis, M.L., A.T., and M.A.A.; investigation, M.L., A.T., M.A.A., and E.D.E.; resources, E.D.E.; data curation, M.L., A.T., and M.A.A.; writing—original draft preparation, M.L., M.A.A., F.D.V., and E.D.E.; writing-review and editing, M.L., A.T., M.A.A., F.D.V., and E.D.E.; visualization, M.L., M.A.A., F.D.V., C.-T.T., and E.D.E.; supervision, C.-T.T., F.D.V., and E.D.E.; project administration, E.D.E.; funding acquisition, C.-T.T., F.D.V., and E.D.E. All authors have read and agreed to the published version of the manuscript.
\end{abstract}

Funding: This research was supported in part by a seed grant from the College of Engineering at FAU and I-SENSE. Research reported in this publication was supported by the National Institute of Biomedical Imaging and Bioengineering of the National Institutes of Health under Award Number R01EB025819. This research was also supported by the National Institute of Aging under 3R01EB025819-04S1 and National Science Foundation awards \#1317952,\#1536136, and \#1950400. This research was also supported by the Boca Raton Regional Hospital Foundation (award \# SP 19-579). The content is solely the responsibility of the authors and does not necessarily represent the official views of the National Institutes of Health or the National Science Foundation.

Institutional Review Board Statement: Not applicable.

Informed Consent Statement: Not applicable.

Data Availability Statement: The data presented in this paper will be made available upon reasonable request.

Acknowledgments: The authors would like to thank R. Paul, W. Cheng, and J. Szalas for their assistance with the soft magnetic sensor array.

Conflicts of Interest: The authors report no conflict of interest concerning the materials or methods used in this study or the findings specified in this paper.

\title{
References
}

1. Todd, A.G. Cervical spine: Degenerative conditions. Curr. Rev. Musculoskelet. Med. 2011, 4, 168. [CrossRef]

2. $\quad$ DiCesare, J.A.; Tucker, A.M.; Say, I.; Patel, K.; Lanman, T.H.; Coufal, F.J.; Millard, J.; Deckey, J.E.; Shetgeri, S.; McBride, D.Q. Mechanical failure of the Mobi-C implant for artificial cervical disc replacement: Report of 4 cases. J. Neurosurg. Spine 2020, 33, 727-733. [CrossRef]

3. Goffin, J.; Casey, A.; Kehr, P.; Liebig, K.; Lind, B.; Logroscino, C.; Pointillart, V.; Van Calenbergh, F.; van Loon, J. Preliminary clinical experience with the Bryan cervical disc prosthesis. Neurosurgery 2002, 51, 840-847. [CrossRef]

4. Bashkuev, M.; Vergroesen, P.-P.A.; Dreischarf, M.; Schilling, C.; van der Veen, A.J.; Schmidt, H.; Kingma, I. Intradiscal pressure measurements: A challenge or a routine? J. Biomech. 2016, 49, 864-868. [CrossRef] [PubMed]

5. Hwang, D.; Gabai, A.S.; Yu, M.; Yew, A.G.; Hsieh, A.H. Role of load history in intervertebral disc mechanics and intradiscal pressure generation. Biomech. Model. Mechanobiol. 2012, 11, 95-106. [CrossRef]

6. Lee, J.K.; Desmoulin, G.T.; Khan, A.H.; Park, E.J. Comparison of 3D spinal motions during stair-climbing between individuals with and without low back pain. Gait Posture 2011, 34, 222-226. [CrossRef] [PubMed]

7. Stollenwerk, K.; Müller, J.; Hinkenjann, A.; Krüger, B. Analyzing Spinal Shape Changes During Posture Training Using a Wearable Device. Sensors 2019, 19, 3625. [CrossRef] [PubMed]

8. Lin, M.; Vatani, M.; Choi, J.-W.; Dilibal, S.; Engeberg, E.D. Compliant underwater manipulator with integrated tactile sensor for nonlinear force feedback control of an SMA actuation system. Sens. Actuators A Phys. 2020, 315, 112221. [CrossRef] [PubMed]

9. Abd, M.A.; Al-Saidi, M.; Lin, M.; Liddle, G.; Mondal, K.; Engeberg, E.D. Surface Feature Recognition and Grasped Object Slip Prevention With a Liquid Metal Tactile Sensor for a Prosthetic Hand. In Proceedings of the 2020 8th IEEE RAS/EMBS International Conference for Biomedical Robotics and Biomechatronics (BioRob), New York, NY, USA, 29 November-1 December 2020; pp. 1174-1179.

10. Liao, X.; Wang, W.; Lin, M.; Li, M.; Wu, H.; Zheng, Y. Hierarchically distributed microstructure design of haptic sensors for personalized fingertip mechanosensational manipulation. Mater. Horiz. 2018, 5, 920-931. [CrossRef]

11. Clifton, W.; Damon, A.; Soares, C.; Nottmeier, E.; Pichelmann, M. Investigation of a three-dimensional printed dynamic cervical spine model for anatomy and physiology education. Clin. Anat. 2021, 34, 30-39. [CrossRef]

12. Parr, W.C.; Burnard, J.L.; Wilson, P.J.; Mobbs, R.J. 3D printed anatomical (bio) models in spine surgery: Clinical benefits and value to health care providers. J. Spine Surg. 2019, 5, 549-560. [CrossRef] [PubMed]

13. Boutry, C.M.; Nguyen, A.; Lawal, Q.O.; Chortos, A.; Rondeau-Gagné, S.; Bao, Z. A sensitive and biodegradable pressure sensor array for cardiovascular monitoring. Adv. Mater. 2015, 27, 6954-6961. [CrossRef] 
14. Chen, D.; Cai, Y.; Huang, M.-C. Customizable pressure sensor array: Design and evaluation. IEEE Sens. J. 2018, 18, 6337-6344. [CrossRef]

15. Almansouri, A.S.; Alsharif, N.A.; Khan, M.A.; Swanepoel, L.; Kaidarova, A.; Salama, K.N.; Kosel, J. An imperceptible magnetic skin. Adv. Mater. Technol. 2019, 4, 1900493. [CrossRef]

16. Mohammadi, A.; Xu, Y.; Tan, Y.; Choong, P.; Oetomo, D. Magnetic-based soft tactile sensors with deformable continuous force transfer medium for resolving contact locations in robotic grasping and manipulation. Sensors 2019, 19, 4925. [CrossRef]

17. Bermúdez, G.S.C.; Makarov, D. Magnetosensitive E-Skins for Interactive Devices. Adv. Funct. Mater. 2021, 31, 2007788. [CrossRef]

18. Bartel, T. Uncertainty in NIST force measurements. J. Res. Natl. Inst. Stand. Technol. 2005, 110, 589. [CrossRef]

19. Chen, D.; Chen, C.-H.; Tang, L.; Wang, K.; Li, Y.-Z.; Phan, K.; Wu, A.-M. Three-dimensional reconstructions in spine and screw trajectory simulation on 3D digital images: A step by step approach by using Mimics software. J. Spine Surg. 2017, 3, 650-656. [CrossRef]

20. Feng, Z.-H.; Li, X.-B.; Phan, K.; Hu, Z.-C.; Zhang, K.; Zhao, J.; Ni, W.-F.; Wu, A.-M. Design of a 3D navigation template to guide the screw trajectory in spine: A step-by-step approach using Mimics and 3-Matic software. J. Spine Surg. 2018, 4, 645-653. [CrossRef]

21. Tyndyk, M.; Barron, V.; McHugh, P.; O'Mahoney, D. Generation of a finite element model of the thoracolumbar spine. Acta Bioeng. Biomech. 2007, 9, 35.

22. Coombs, D.; Rao, M.; Bushelow, M.; Deacy, J.; Laz, P.; Rullkoetter, P. Simulation of lumbar spine biomechanics using Abaqus. In Proceedings of the SIMULIA Customer Conference, Barcelona, Spain, 17-19 May 2011.

23. Song, M.; Zhang, Z.; Lu, M.; Zong, J.; Dong, C.; Ma, K.; Wang, S. Four lateral mass screw fixation techniques in lower cervical spine following laminectomy: A finite element analysis study of stress distribution. Biomed. Eng. Online 2014, 13, 115. [CrossRef] [PubMed]

24. DiAngelo, D.J.; Foley, K.T.; Morrow, B.R.; Schwab, J.S.; Song, J.; German, J.W.; Blair, E. In vitro biomechanics of cervical disc arthroplasty with the ProDisc-C total disc implant. Neurosurg. Focus 2004, 17, 44-54. [CrossRef]

25. Abd, M.A.; Paul, R.; Aravelli, A.; Bai, O.; Lagos, L.; Lin, M.; Engeberg, E.D. Hierarchical Tactile Sensation Integration from Prosthetic Fingertips Enables Multi-Texture Surface Recognition. Sensors 2021, 21, 4324. [CrossRef]

26. Cortes, C.; Vapnik, V. Support-vector networks. Mach. Learn. 1995, 20, 273-297. [CrossRef]

27. Fix, E.; Hodges, J.L. Discriminatory analysis. Nonparametric discrimination: Consistency properties. Int. Stat. Rev. /Rev. Int. De Stat. 1989, 57, 238-247. [CrossRef]

28. Breiman, L. Random forests. Mach. Learn. 2001, 45, 5-32. [CrossRef]

29. Fitch, F.B.; McCulloch Warren, S.; Walter, P. A logical calculus of the ideas immanent in nervous activity. Bulletin of mathematical biophysics, vol. 5, pp. 115-133. J. Symb. Log. 1944, 9, 49-50. [CrossRef]

30. Dindorf, C.; Konradi, J.; Wolf, C.; Taetz, B.; Bleser, G.; Huthwelker, J.; Werthmann, F.; Bartaguiz, E.; Kniepert, J.; Drees, P.; et al Classification and Automated Interpretation of Spinal Posture Data Using a Pathology-Independent Classifier and Explainable Artificial Intelligence (XAI). Sensors 2021, 21, 6323. [CrossRef]

31. Patwardhan, A.G.; Havey, R.M.; Ghanayem, A.J.; Diener, H.; Meade, K.P.; Dunlap, B.; Hodges, S.D. Load-carrying capacity of the human cervical spine in compression is increased under a follower load. Spine 2000, 25, 1548-1554. [CrossRef]

32. Pospiech, J.; Stolke, D.; Wilke, H.J.; Claes, L.E. Intradiscal pressure recordings in the cervical spine. Neurosurgery 1999, 44, 379-384 [CrossRef]

33. Galbusera, F.; Bellini, C.M.; Zweig, T.; Ferguson, S.; Raimondi, M.T.; Lamartina, C.; Brayda-Bruno, M.; Fornari, M. Design concepts in lumbar total disc arthroplasty. Eur. Spine J. 2008, 17, 1635-1650. [CrossRef] [PubMed]

34. Lotz, J.C. Animal models of intervertebral disc degeneration: Lessons learned. Spine 2004, 29, 2742-2750. [CrossRef] [PubMed]

35. Khan, M.H.; Rihn, J.; Steele, G.; Davis, R.; Donaldson III, W.F.; Kang, J.D.; Lee, J.Y. Postoperative management protocol for incidental dural tears during degenerative lumbar spine surgery: A review of 3183 consecutive degenerative lumbar cases. Spine 2006, 31, 2609-2613. [CrossRef]

36. Accardi-Ravid, M.; Eaton, L.; Meins, A.; Godfrey, D.; Gordon, D.; Lesnik, I.; Doorenbos, A. A qualitative descriptive Study of patient experiences of pain before and after spine surgery. Pain Med. 2020, 21, 604-612. [CrossRef] [PubMed]

37. Mallow, G.M.; Siyaji, Z.K.; Galbusera, F.; Espinoza-Orías, A.A.; Giers, M.; Lundberg, H.; Ames, C.; Karppinen, J.; Louie, P.K.; Phillips, F.M.; et al. Intelligence-based spine care model: A new era of research and clinical decision-making. Glob. Spine J. 2021, 11, 135-145. [CrossRef] [PubMed]

38. Lin, M.; Shapiro, S.Z.; Doulgeris, J.; Engeberg, E.D.; Tsai, C.-T.; Vrionis, F.D. Cage-screw and anterior plating combination reduces the risk of micromotion and subsidence in multilevel anterior cervical discectomy and fusion-A finite element study. Spine J. 2021, 21, 874-882. [CrossRef]

39. Abbed, K.M.; Coumans, J.-V.C. Cervical radiculopathy: Pathophysiology, presentation, and clinical evaluation. Neurosurgery 2007, 60, S1-28-S21-34. [CrossRef]

40. Diebo, B.G.; Ferrero, E.; Lafage, R.; Challier, V.; Liabaud, B.; Liu, S.; Vital, J.-M.; Errico, T.J.; Schwab, F.J.; Lafage, V. Recruitment of compensatory mechanisms in sagittal spinal malalignment is age and regional deformity dependent: A full-standing axis analysis of key radiographical parameters. Spine 2015, 40, 642-649. [CrossRef]

41. Paladugu, P.; Hernandez, A.; Gross, K.; Su, Y.; Neseli, A.; Gombatto, S.; Moon, K.; Ozturk, Y. A sensor cluster to monitor body kinematics. In Proceedings of the 2016 IEEE 13th International Conference on Wearable and Implantable Body Sensor Networks (BSN), San Francisco, CA, USA, 14-17 June 2016; pp. 212-217. 
42. Hannink, E.; Shannon, T.; Barker, K.L.; Dawes, H. The reliability and reproducibility of sagittal spinal curvature measurement using the Microsoft Kinect V2. J. Back Musculoskelet. Rehabil. 2020, 33, 295-301. [CrossRef] 